\title{
Frontières
}

\section{Ascension, apothéose et morbidité du phénomène urbain}

\section{Luc-Normand Tellier}

Volume 15, numéro 1, automne 2002

Délires urbains, dangers de mort

URI : https://id.erudit.org/iderudit/1073900ar

DOI : https://doi.org/10.7202/1073900ar

Aller au sommaire du numéro

Éditeur(s)

Université du Québec à Montréal

ISSN

1180-3479 (imprimé)

1916-0976 (numérique)

Découvrir la revue

Citer cet article

Tellier, L.-N. (2002). Ascension, apothéose et morbidité du phénomène urbain. Frontières, 15(1), 7-14. https://doi.org/10.7202/1073900ar

\section{Résumé de l'article}

Les premières villes urbaines apparaissent dans la région de Sumer, il y a 5500 ans environ. À compter de cette époque, au cours de la Première révolution urbaine, il aura fallu environ 5325 ans pour que le taux d'urbanisation mondial atteigne $5 \%$. En 1825, les transports motorisés font leur apparition. Entre cette date et aujourd'hui, au cours de la Seconde révolution urbaine, le taux d'urbanisation mondial est passé de $5 \%$ à près de $50 \%$. Cette polarisation croissante des populations s'est accompagnée d'une polarisation encore plus prononcée du pouvoir économique. L’urbanisation actuelle est porteuse de plusieurs maux, qui diffèrent suivant les niveaux de développement, mais aussi de nombreux espoirs de naissance d'une urbanité nouvelle. 


\section{Résumé}

Les premières villes urbaines apparaissent dans la région de Sumer, il y a 5500 ans environ. À compter de cette époque, au cours de la Première révolution urbaine, il aura fallu environ 5325 ans pour que le taux d'urbanisation mondial atteigne $5 \%$. En 1825 , les transports motorisés font leur apparition. Entre cette date et aujourd'hui, au cours de la Seconde révolution urbaine, le taux d'urbanisation mondial est passé de $5 \%$ à près de $50 \%$. Cette polarisation croissante des populations s'est accompagnée d'une polarisation encore plus prononcée du pouvoir économique. L'urbanisation actuelle est porteuse de plusieurs maux, qui diffèrent suivant les niveaux de développement, mais aussi de nombreux espoirs de naissance d'une urbanité nouvelle.

Mots clés : phénomène urbain - mort vie-morbidité - étalement urbain

\section{Abstract}

Roughly 5,550 years ago the first urban cities sprung up in the Sumer Region. From this period, during the First Urban Revolution, it would take some 5,325 years for the world urbanization rate to reach $5 \%$. In 1825, motorized transportation arrived on the scene. Between that date and today, during the Second Urban Revolution, the world urbanization rate rose from $5 \%$ to nearly $50 \%$. This growing population polarization is coupled with an even more pronounced polarization of economic power. Current urbanization bears many pitfalls, which vary according to development levels, yet also raises much hope of the emergence of a new urbanity.

Key words : urban phenomenon - deathlife-morbidity-urban sprawl

\section{ASCENSION, APOTHÉOSE ET MORBIDITÉ DU PHÉNOMĖNE URBAIN}

\begin{abstract}
Luc-Normand Tellier, Ph.D., professeur, Département d'études urbaines et touristiques, UQÀM.
\end{abstract}

Le monde moderne est à ce point urbain que nos contemporains ont le plus souvent tendance à tenir le phénomène urbain pour acquis et à croire que, depuis toujours, il y a eu des villes et que l'être humain est, pour ainsi dire, né urbain. Pourtant, rien n'est plus loin de la vérité. Le phénomène urbain est un phénomène tout récent dans l'histoire plurimillénaire de l'homo sapiens sapiens. Si ce dernier est apparu il y a environ 150000 ans, les premières «villes urbaines » ne font leur apparition dans la région de Sumer que vers 4300-3100 avant Jésus-Christ, soit il y a environ 5600 ans. Donc, pendant $96 \%$ de son existence, l'homo sapiens sapiens que nous sommes n'a rien eu d'urbain. Bien plus, il y a tout juste 200 ans, vers 1800, le taux d'urbanisation mondial se situait sans doute seulement autour de $5 \%$, ce qui est fort peu (il a atteint 29,4\% en 1950 et 48,2\% en 2000). Ce n'est qu'au début du troisième millénaire que la majorité de la population mondiale sera urbaine.

Nous nous attarderons ici à reconstituer l'histoire du phénomène urbain afin de faire ressortir le contraste frappant entre le triomphe récent et même brutal des tendances à l'urbanisation et l'affaiblissement encore plus récent de l'intégration intraurbaine, affaiblissement lié à l'étalement urbain et générateur d'une nouvelle «morbidité » du phénomène urbain. Le mot morbidité doit ici être entendu dans son sens propre, soit celui de l' " ensemble des causes qui peuvent produire une maladie », ce qui est distinct de la maladie elle-même. Si le phénomène urbain peut être associé à divers maux, ces maux ne lui sont nullement indissociablement liés, tout comme la ville est tout autant porteuse d'espoirs et de potentialités que de problèmes.

\section{LA PREMIÈRE RÉVOLUTION URBAINE : DE CCATAL HÖYÜK ET ÉRIDOU À 1825}

L'apparition des premières villes « urbaines » a été précédée par celle des premières villes " préurbaines », la différence entre les unes et les autres tenant au fait qu'une agglomération d'importance ne peut revêtir un caractère véritablement urbain que dans le contexte de l'existence d'un véritable réseau urbain donnant lieu à des échanges commerciaux interurbains ${ }^{1}$. Les trois villes «préurbaines » les plus célèbres sont Çatal Höyük, en Anatolie (Turquie), Jéricho, en Palestine, et Jarmo, dans la région du haut Tigre (Irak). La plus vieille et la plus grosse d'entre elles est Çatal Höyük dont l'apparition remonte à 9000 ans et peut-être plus. On estime que, vers 7000 avant Jésus-Christ, cette ville comptait environ 6000 habitants.

Çatal Höyük avait la particularité de ne contenir ni rue ni ruelle; les habitants de la ville passaient d'une habitation à une 


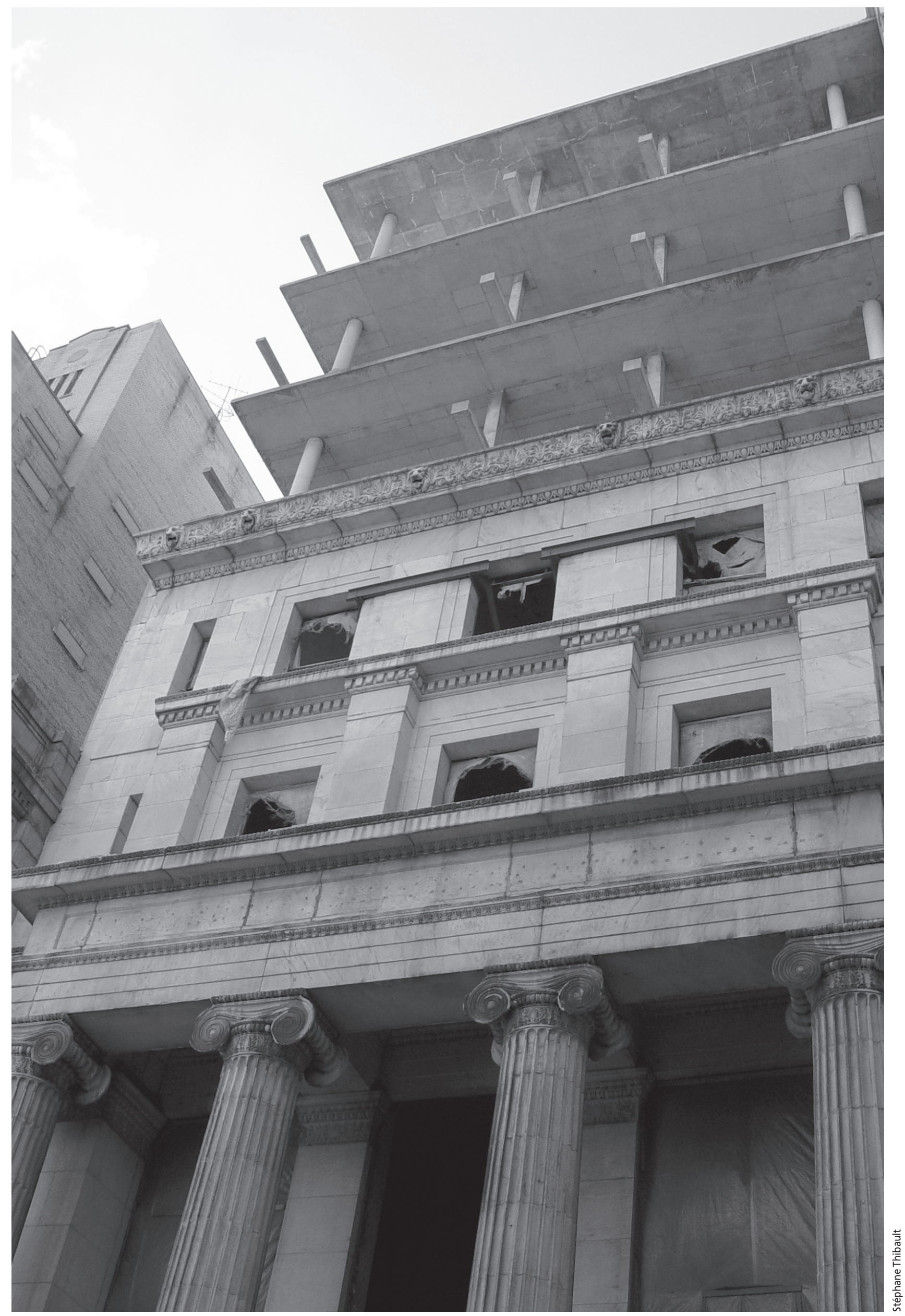


autre en marchant sur les toits auxquels ils accédaient par des échelles. Cette caractéristique assez étrange disparaîtra très rapidement dès le début de l'histoire des villes. Cependant, dès l'apparition de Çatal Höyük, le schéma le plus marquant de toute l'histoire de l'urbanisation vit le jour.

Celui-là est caractérisé par la formation d'un noyau central entouré par une première périphérie, puis par une seconde périphérie. Dans le cas de Çatal Höyük, le noyau central est constitué de la ville elle-
Ni Çatal Höyük, ni Jéricho, ni Jarmo ne faisaient partie d'un système urbain. Elles étaient trop isolées et aucune voie de communication ne les reliait à d'autres villes. Le premier véritable système urbain constitué de villes « urbaines » reliées entre elles par des réseaux et des échanges est apparu en Mésopotamie dans la région de Sumer vers 3500 avant Jésus-Christ avec l'émergence des villes d'Éridou, Our, Ourouk et leurs voisines. L'apparition de ce premier noyau urbain fut suivie, dans l'ordre, de

\section{LA VILLE EST TOUT AUTANT PORTEUSE D'ESPOIRS}

\section{ET DE POTENTIALITÉS QUE DE PROBLÈMES.}

même où apparaissent les premières fonctions de fabrication et de gestion. Quant à la première périphérie (ou semi-périphérie, pour reprendre l'expression de Braudel), celle du foyer égyptien (avec Memphis et Thèbes), du centre urbain de la vallée de l'Indus (avec Harappa, Mohenjo-daro et Kalibangan), puis du berceau urbain de la

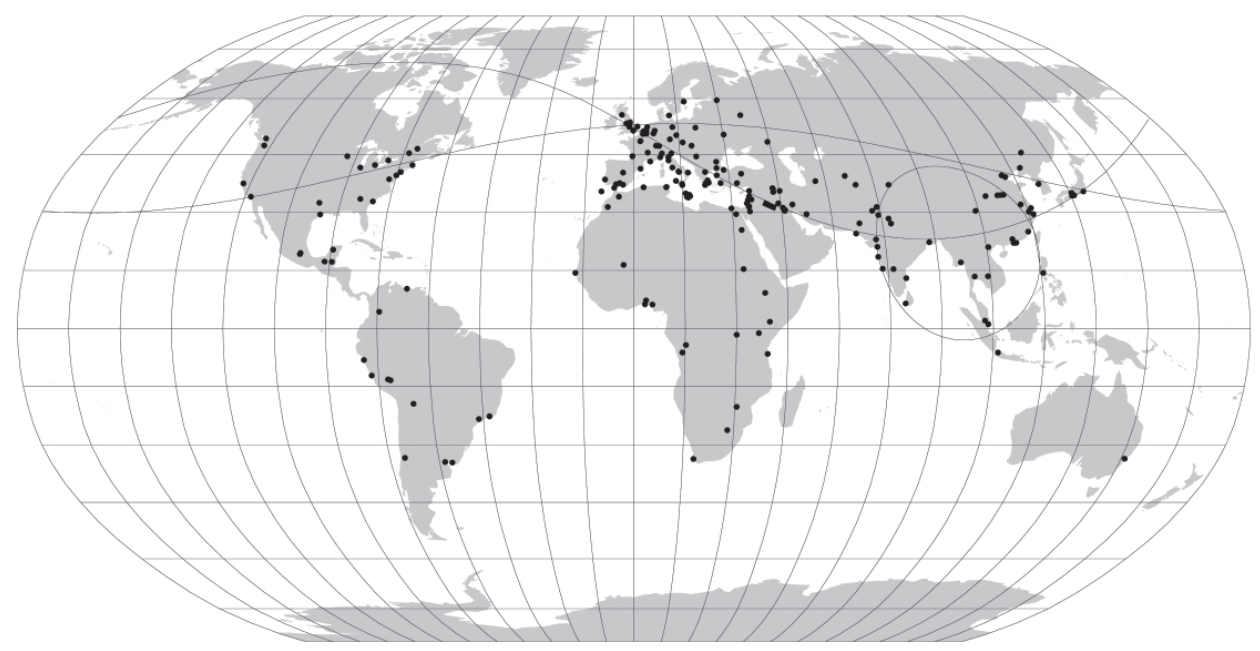

elle est marquée par la mise en valeur des ressources (dans le cas de Çatal Höyük, par l'agriculture et par l'élevage). Enfin, une seconde périphérie existe, tentaculaire et caractérisée par le prélèvement pur et simple des ressources (dans le cas de Çatal Höyük, par la pêche, la chasse, la cueillette ou l'extraction de métaux).

Ce même schéma "noyau central spécialisé dans la fabrication et la gestion semi-périphérie spécialisée dans la mise en valeur des ressources - périphérie tentaculaire spécialisée dans le prélèvement des ressources» se retrouve tout au long de l'histoire urbaine tant au niveau des villes prises séparément qu'au niveau des systèmes urbains tout entiers ${ }^{2}$. Il caractérise même aujourd'hui le système urbain mondial dans son ensemble, lequel comporte trois noyaux centraux dominés respectivement par New York, Londres et Tokyo.
Chine (avec Changan-Sian-Xi'an, LoyangLuoyang, Chengchow-Zhengzhou et Kaifeng).

La première révolution urbaine a été précédée par l'apparition de l'agriculture plus d'un millénaire plus tôt, mais elle a vraisemblablement été déclenchée par des innovations liées aux communications qui sont apparues à peu près au même moment que les premières villes « urbaines » : inventions de l'écriture, de la roue, du chariot et des premières embarcations plus perfectionnées que le simple radeau. Il y a tout lieu de croire que la première révolution urbaine a été causée par un changement radical dans les conditions de la mobilité tout comme la seconde révolution urbaine a été provoquée par le passage du transport animal au transport motorisé.

Dès l'origine, les villes, les systèmes urbains et les réseaux de communication se sont trouvés en concurrence. Dans la région du Croissant fertile, trois axes se sont disputé la suprématie: l'axe de l'Euphrate, celui du Tigre et celui du Nil. L'empire égyptien a tablé sur le Nil, l'empire assyrien, sur le Tigre et les empires babyloniens, sur l'Euphrate. Avec les triomphes du second empire babylonien de Nabuchodonosor II, puis de l'empire perse et de l'empire d'Alexandre le Grand, c'est l'axe de l'Euphrate qui s'est imposé grâce à la jonction que le Couloir syrien a permis d'établir entre l'Euphrate et la Méditerranée.

Cet axe est une des sections les plus importantes du Grand corridor ${ }^{3}$ et le mouvement de Sumer vers la Méditerranée est à l'origine du grand mouvement vers l'ouest à l'intérieur du Grand corridor, mouvement qui a marqué la naissance de l'Occident. Cette trajectoire est allée de Sumer vers Babylone, la Phénicie, l'Anatolie et la Grèce, puis vers l'Italie et la Gaule. Elle s'est accompagnée d'un mouvement d'urbanisation (qu'avait précédé plus d'un millénaire plus tôt un mouvement de diffusion de l'agriculture) ainsi que d'un mouvement de développement économique, social et culturel.

Le Grand corridor correspond à la limite sud de la "chaîne de chaînes de montagnes» qui domine le continent euroasiatique. D'est en ouest, cette chaîne comprend le Plateau du Yunnan (Chine), l'Himalaya, les monts Karakorum (Cachemire), les monts Souleiman (Pakistan), le Plateau d'Iran, les monts Zagros (Iran), les monts Taurus (Turquie), les Balkans et les Alpes. De nombreux cours d'eau prennent leur source dans ces montagnes : le Yangzi jiang, le Tongkiang (rivière des Perles), le Gange, l'Indus, le Tigre, l'Euphrate, le Pô, le Rhône et le Rhin. Tous ces fleuves à vocation économique constituent, avec la Méditerranée, le golfe Persique et la mer d'Oman, une chaîne qui sert de colonne vertébrale au Grand corridor. Ensemble, ils canalisent les grands courants commerciaux qui irriguent le Grand corridor à l'intérieur duquel, depuis 5000 ans, se sont succédé la majorité des principaux pôles économiques du monde: Babylone, Alexandrie, Athènes, Rome, Constantinople, Antioche, Bagdad, Venise, Gênes, Anvers, Amsterdam, Londres, Paris, tout comme Delhi, Canton-Guangzhou, Zayton-ChuanchowQuanzhou, Shanghai et Tokyo.

Jusqu'à la fondation de Constantinople, en 330 après Jésus-Christ, la tendance vers l'ouest a nettement prévalu à l'ouest du golfe Persique, tandis que la tendance vers l'est l'emportait à l'est du golfe, où, grosso modo, le développement de la Perse a précédé celui de l'Inde, celui de l'Inde a précédé celui de la Chine, celui de la Chine a précédé celui de la Corée et celui de la Corée a précédé celui du Japon. 
Le mouvement vers l'ouest a atteint une limite ultime quand l'empire romain a atteint l'Angleterre et l'Atlantique qui, avant la découverte de l'Amérique, était un véritable cul-de-sac. La montée de Constantinople et la chute de Rome en 476 après Jésus-Christ ont marqué le renversement relativement brutal de la tendance vers l'ouest. La nouvelle tendance vers l'est a rapidement, à compter de 632 (mort de Mahomet), pris la couleur de l'Islam. Après être passée de Rome à Constantinople, la puissance économique et politique passa à Damas, au Caire et à Bagdad. L'impact de ce Grand Ressac sur l'urbanisation a été radical. Le taux d'urbanisation de l'Europe occidentale est alors tombé d'environ $6 \%$ à $0 \%$ : une catastrophe !

Un second corridor a aussi joué un rôle dans l'histoire du développement économique et de l'urbanisation. Il s'agit du Corridor asiatique qui correspond aux routes continentale et maritime de la Soie. Ce corridor est issu de l'apparition de l'urbanisation dans la vallée de l'Indus. Avant la révolution industrielle, les tendances prédominantes à l'intérieur de ce corridor ont été d'ouest en est. Dans la partie nord du Corridor, dans la partie correspondant à la route continentale de la Soie, l'urbanisation de la vallée de l'Indus a été suivie de l'émergence de l'urbanisation en Chine dans la région de Changan-Sian-Xi'an, puis, au VIII ${ }^{\mathrm{e}}$ siècle après Jésus-Christ, du transfert de la puissance économique de la région du lœss basée sur la culture du blé et du millet à la région du Yangzi jiang basée sur la culture du riz. Cette trajectoire économique correspond en grande partie à celle de la diffusion du bouddhisme, puis, plus tard, à celle de l'expansion de l'Islam, dans le contexte du Grand Ressac dont les répercussions se sont aussi fait sentir dans le Corridor asiatique.

Dans la partie sud du Corridor asiatique, à la suite du Grand Ressac, le mouvement fut fortement marqué par la diffusion de l'Islam qui gagna le Pakistan, puis l'Inde actuels, et, ensuite, la Malaisie, l'Indonésie et même les Philippines ; ce, grâce aux commerçants arabes qui furent les premiers commerçants étrangers à s'établir en Chine, à Canton-Guangzhou et le long du Tongkiang (rivière des Perles).

Lors de la conquête de Constantinople par les Turcs en 1453, l'Europe chrétienne était menacée de toutes parts. La Russie demeurait sous la suzeraineté des Mongols musulmans de la Horde d'Or (Ivan III le Grand ne se libéra de la suzeraineté mongole qu'en 1480). L'empire byzantin s'écroulait et les musulmans étaient toujours présents en Espagne (Grenade ne tomba qu'en 1492). Cependant, depuis le déclenchement des croisades par Urbain II en 1095, l'Occident avait résolu d'affronter la menace musulmane. Si les défaites des Croisés ont été plus nombreuses que leurs victoires, dans la péninsule ibérique, la Reconquista progressait: Tolède avait été reconquise en 1085, Lisbonne, en 1147, et Séville, en 1248. Bien plus, le rêve était caressé, surtout au Portugal (par Henri le Navigateur), de contourner le monde musulman par la mer afin de renouer les liens commerciaux entre l'Occident et l'Inde et les liens religieux entre l'Europe catholique et l'Éthiopie chrétienne. Cela conduisit directement à la découverte de l'Amérique par Christophe Colomb, en 1492, et à la découverte, en 1497, par Vasco de Gama, de la route de l'Inde contournant l'Afrique par le sud.

Ces événements eurent autant de conséquences que la chute de Rome. Ils relancèrent le mouvement vers l'ouest que la chute de l'empire romain d'Occident avait interrompu. Le pouvoir économique passa alors successivement de Constantinople à Venise et Gênes, puis, au XVII ${ }^{\mathrm{e}}$ siècle, à Anvers (Brabant), Amsterdam (Hollande) et, finalement, Londres ${ }^{4}$.
Pendant toute la longue période de la première révolution urbaine, l'urbanisation a été le produit de modes de transport tributaires, sur terre, de la traction animale et, sur la mer, de la voile ou de la rame. Compte tenu de ces conditions de la mobilité, les villes étaient partout compactes au point d'être le plus souvent entourées de murailles.

Certaines évolutions se sont néanmoins produites pendant ces 5000 à 5500 années. Aux villes refuges et aux villes oasis ont progressivement succédé des villes fluviales de plus en plus axées sur le commerce, puis des villes maritimes. Cette évolution a été observée autant au Moyen-Orient où les villes phéniciennes, grecques et italiennes de la Méditerranée ont pris le relais des villes fluviales de la Mésopotamie, et en Chine où les villes fluviales de la région du lœss ont progressivement été concurrencées par des villes à vocation maritime (CantonGuangzhou, Zayton-Chuanchow-Quanzhou et Hangchow-Hangzhou). Parallèlement, aux empires continentaux (empires babylonien, assyrien, égyptien, hittite, perse et d'Alexandre le Grand) ont succédé les réseaux maritimes phéniciens et grecs, puis le premier véritable empire maritime, l'empire romain. Plus tard, à la suite de la découverte de l'Amérique, aux empires maritimes locaux (comme celui de Venise et celui de Gênes) succédèrent les empires mondiaux : portugais, espagnol, hollandais, français et anglais.

\section{LA SECONDE RÉVOLUTION URBAINE}

C'est alors que se produisit la seconde révolution urbaine avec l'apparition du transport motorisé. La motorisation a d'abord touché la navigation (Robert Fulton lança le premier navire à vapeur qui fit le trajet de New York à Albany, en août 1807). C'est cependant l'inauguration, le 27 septembre 1825, du premier chemin de fer

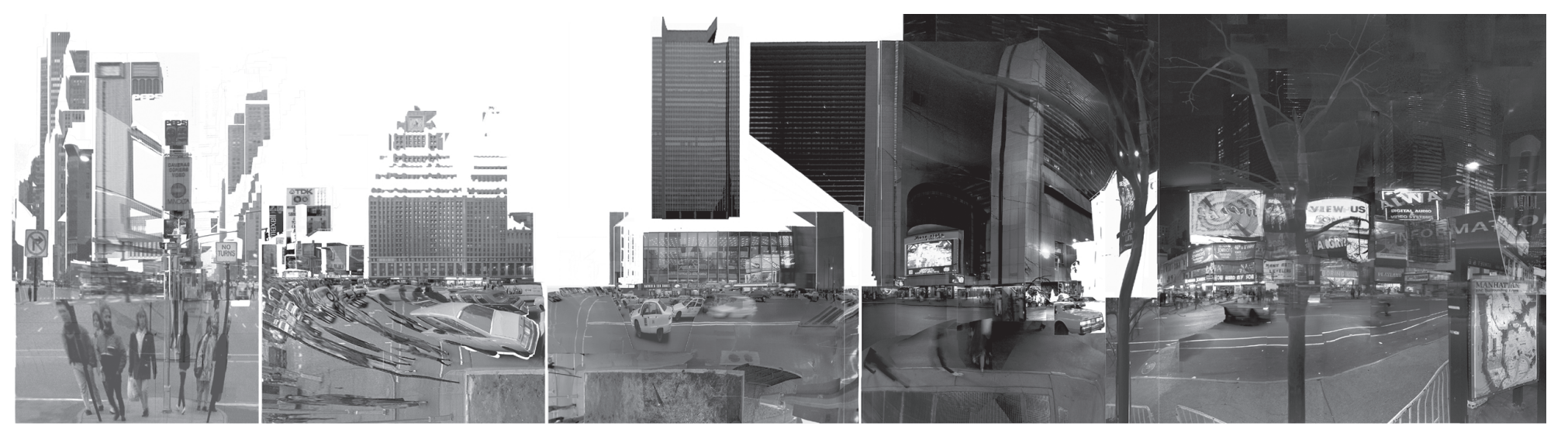


entre Stockton et Darlington en Angleterre, au centre de l'empire britannique triomphant, qui marqua le véritable début de la seconde révolution urbaine qui fit passer le taux d'urbanisation mondial d'environ $5 \%$ en 1825 à 48,2\% en 2000.

Pour bien saisir l'impact de l'avènement du transport motorisé, il convient de dire que la révolution industrielle associée à ce dernier a été précédée en Angleterre par ce qu'on a appelé la «protoindustrialisation » au cours de laquelle de nombreuses innovations techniques ont été implantées. Or la protoindustrialisation s'est faite avant tout dans les campagnes et elle semble s'être traduite par une légère baisse de l'urbanisation, alors que l'industrialisation marquée par le transport motorisé eut l'effet inverse. Elle provoqua une véritable explosion urbaine qui, en Grande-Bretagne, se traduisit par le triomphe de nouvelles villes (Leeds, Liverpool, Manchester, Glasgow), tandis qu'en France, les vieilles villes (Lille, Lyon, Saint-Étienne) réussirent à s'adapter à l'industrialisation sans être déclassées.

L'épicentre de la seconde révolution urbaine a été Londres à partir de laquelle l'onde de choc de l'industrialisation se propagea à l'intérieur du Grand Corridor en touchant d'abord la Belgique et la France, puis en atteignant, à l'intérieur d'un nouveau corridor, vers l'ouest, les États-Unis et le Canada, et, vers l'est, l'Allemagne, la Silésie et même la Russie. Ce corridor, nommé Corridor américain, est un pur produit des nouvelles infrastructures de transport associées à l'industrialisation : soit les canaux et les chemins de fer.

En Amérique du Nord, il est structuré par le canal Érié inauguré en 1825 (ce canal a assuré l'ascendant de New York sur le reste du continent) et par l'axe ferroviaire transcontinental reliant New York, Pittsburgh, Cincinnati, Saint Louis, Kansas City et Los Angeles. Le centre de gravité des populations aux États-Unis suit précisément cette trajectoire depuis 1790 jusqu'à aujourd'hui. En Eurasie, le Corridor américain est structuré par le Mittellandkanal, qui relie la vallée de la Ruhr à Berlin, et par l'axe ferroviaire qui va de Berlin vers Varsovie, Moscou, Novosibirsk et Vladivostok, la section Moscou-Vladivostok correspondant au Transsibérien.

L'émergence du Corridor américain a eu un impact majeur tant en Amérique qu'en Eurasie. Elle a provoqué une urbanisation fulgurante de l'ensemble canado-américain qui avait un retard considérable sur l'Amérique latine qui avait déjà connu l'urbanisation sous les Mayas, les Aztèques et les Incas. L'Amérique latine était déjà, en 1825, une des régions les plus urbanisées du monde, alors que le Canada et les ÉtatsUnis demeuraient fort peu urbanisés.

En Eurasie, la montée du Corridor américain s'est traduite par le triomphe de la Prusse sur l'Autriche (qui fait partie du Grand corridor) dans le monde germanique, puis par celui de l'Empire russe sur l'Empire ottoman. La montée du Japon s'inscrit dans le mouvement vers l'ouest à l'intérieur du Corridor américain. C'est en effet sous la pression des Américains que le Japon a dû accepter de s'ouvrir à compter de 1854 , ce qui a été suivi, en 1868, par le début de l'ère Meiji au cours de laquelle le Japon s'est radicalement modernisé. Les deux trajectoires (vers l'ouest et vers l'est) sont entrées en collision en 1905, lors du conflit russo-japonais, lorsque le Japon qui avait conquis la Corée, est entré en Mandchourie et a affronté les Russes que l'expansion vers l'est avait aussi conduits en Mandchourie. Notons que la Mandchourie est aux antipodes de New York, à l'intérieur du Corridor américain.

De 1825 à la crise de 1929, le monde a beaucoup changé. Les villes industrielles se sont considérablement développées, le plus souvent au milieu de la pollution, de la promiscuité et des tensions sociales. Les villes ont commencé à s'étendre dans toutes les directions. De nombreuses nouvelles villes sont apparues, plusieurs d'un type nouveau : les villes ferroviaires. Ces dernières et les villes à vocation océanique (comme Londres, New York et Tokyo) l'ont peu à peu emporté sur les villes fluviales qui ne réussissaient pas à s'intégrer au réseau ferroviaire, ou sur les villes simplement maritimes (comme Venise ou Istanbul). Les empires mondiaux se sont considérablement développés; singulièrement, le plus grand d'entre eux, l'Empire britannique dont les ramifications s'étendaient partout, en Afrique, en Asie, en Amérique latine, en Amérique du Nord et en Océanie. Cependant, la montée des États-Unis a fait percevoir que la mondialisation et l'exercice d'un pouvoir à l'échelle du globe étaient aussi possibles sans empire.

\section{TRIOMPHE DE L'AUTOMOBILE ET APOTHÉOSE \\ DE L'URBANISATION}

En 1900, Londres était encore la ville la plus puissante du monde. Cinquante ans plus tard, ce titre revenait incontestablement à New York. Bien plus, entre 1900 et 1950, le Corridor américain a nettement supplanté le Grand Corridor avec la consécration de New York, Chicago, Berlin (jusqu'en 1944), Moscou et Tokyo. Cette période marque aussi le triomphe de l'automobile qui fait pénétrer le transport motorisé dans les moindres recoins du monde développé et même du monde en développement.

Avec l'omniprésence de l'automobile, l'urbanisation atteint son apothéose. Dans les pays développés, des taux d'urbanisation de $75 \%$ et plus sont devenus la norme (le taux d'urbanisation atteint même 95\% en Belgique) et on retrouve dans les pays en développement des taux aussi élevés que $83 \%$ (Venezuela) ou $73 \%$ (Irak). Au cours $\mathrm{du} \mathrm{XX}^{\mathrm{e}}$ siècle, alors que la population du

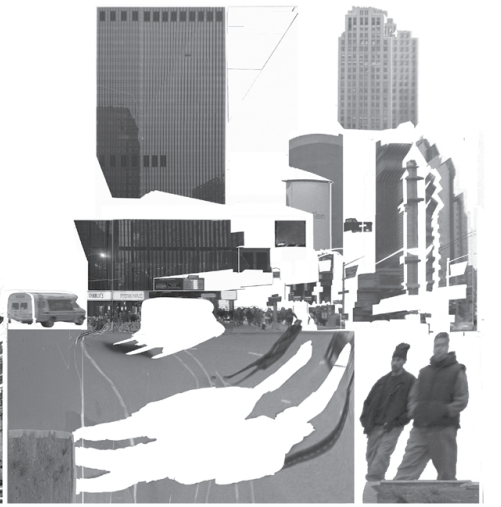


monde était multipliée par 3,6 (passant de 1,65 en 1900 à 6 milliards d'habitants en 2000), celle des villes a été multipliée par 15 (passant de 193 millions à 2,892 milliards). Ce triomphe de l'urbanisation s'accompagne (aussi, en bonne partie, à cause de l'automobile) d'un éclatement de la ville, le phénomène de l'étalement urbain étant aussi universel que l'urbanisation elle-même.

Partout le monde rural bat en retraite. Même les villes à vocation rurale connaissent un déclin relatif, tandis que les grandes villes se tertiarisent à toute vitesse. Même le secteur secondaire (secteur de la transformation), qui dominait les villes de la révolution industrielle, se voit relégué au second plan dans les villes des pays développés.

\section{QUAND L'URBANISATION CESSE D'ÊTRE SYNONYME DE DÉVELOPPEMENT}

Le monde est de plus en plus dominé par trois pôles économiques majeurs: le pôle nord-américain centré sur New York (et, de plus en plus, Los Angeles), l'Europe occidentale centrée sur Londres et Paris, et l'Extrême-Orient centré sur Tokyo (et aussi Shanghai et Hongkong). On assiste aujourd'hui à une dissociation de la polarisation des populations et de celle des productions. Les trois pôles économiques correspondent de moins en moins aux plus grandes concentrations de populations, ce qui est un phénomène nouveau dans l'his- est remonté à 19 à 1 en 1998, alors que la région la plus riche correspond aux nouveaux pays occidentaux (États-Unis, Canada, Australie et Nouvelle-Zélande) et la région la plus pauvre est l'Afrique (Maddison, 2001, p. 126). Aujourd'hui, ce ne sont plus les centres de l'économie mondiale (Londres, Paris, New York, Tokyo, Chicago ou Los Angeles) qui connaissent une explosion de population ; ce sont plutôt les métropoles du tiers monde (Mexico, Lagos, Le Caire, São Paulo, Calcutta, Bombay).

L'urbanisation a cessé d'être synonyme de développement, ce qui ne veut pas dire qu'elle soit devenue un obstacle à ce dernier. Cette dissociation (relativement récente) de la polarisation des populations et de celle des productions est le grand phénomène de ce début de millénaire. Il menace peut-être plus l'humanité que l'arme nucléaire ou le réchauffement de la planète. Et pourtant personne n'en parle.

Ce phénomène est associé à un autre phénomène sans précédent dans l'histoire, celui du développement d'une "sur-urbanisation en périphérie ». Le phénomène de sur-urbanisation caractérisé par une polarisation des populations excédant ce qui est souhaitable compte tenu de la polarisation des productions n'est pas un phénomène récent: la Rome antique au $\mathrm{IV}^{\mathrm{e}}$ siècle et une ville comme Naples aux XVIII ${ }^{\mathrm{e}}$ et $\mathrm{XIX}^{\mathrm{e}}$ siècles en sont des exemples classiques. Ce phénomène était alors associé à

ON ASSISTE AUJOURD'HUI À UNE DISSOCIATION DE LA POLARISATION

DES POPULATIONS ET DE CELLE DES PRODUCTIONS. LES TROIS PÔLES ÉCONOMIQUES CORRESPONDENT DE MOINS EN MOINS AUX PLUS GRANDES CONCENTRATIONS DE POPULATIONS, CE QUI EST UN PHÉNOMÈNE NOUVEAU DANS L'HISTOIRE UNIVERSELLE.

toire universelle. En somme, au moment où l'urbanisation devient un phénomène mondial touchant tous les pays du monde et alors qu'un taux d'urbanisation de $60 \%$ et plus sera bientôt la norme partout à travers le monde et que la polarisation des populations est sur le point de plafonner, le développement économique continue, lui, à se polariser de plus en plus et à engendrer des inégalités croissantes. En 1820, le rapport entre le produit par habitant de la région la plus riche du monde (l'Europe occidentale) et de la région la plus pauvre (l'Afrique) était de 3 à 1 . Ce rapport est passé à 15 à 1 en 1950 ; il est descendu à 13 à 1 en 1973 et il des villes dominantes ayant attiré une trop large population et qui connaissaient soudainement un déclin marqué.

La sururbanisation qui est actuellement observée dans les régions du monde économiquement périphériques n'est pas de ce type. Elle est liée au fait que plusieurs villes du tiers monde servent de plus en plus non pas de tremplins, mais bien de refuges, de bouées de sauvetage au milieu de la tempête causée par la polarisation des productions à l'échelle mondiale.

Il faut cependant ajouter immédiatement que la sur-urbanisation n'affecte pas toutes les régions qui faisaient partie de la péri- phérie en 1960-1970, par exemple. Le monde bouge et la périphérie mondiale, aussi. Des zones complètes de l'ancienne périphérie sont aujourd'hui en émergence. Qu'on songe à la Corée du Sud, à la partie orientale de la Chine, à Singapour, à la Malaysia, ou même à l'île Maurice ou au nord du Mexique. Dans toutes ces régions qui montent, l'urbanisation est le plus souvent saine et ne saurait être qualifiée de sur-urbanisation. Il ne faut jamais faire l'erreur d'oublier que des régions pauvres émergent du sousdéveloppement, alors que d'autres, jadis riches, semblent s'y enfoncer (qu'on songe aux cas des ex-républiques soviétiques ou de l'Argentine, dont les situations peuvent cependant être conjoncturelles).

L'opposition traditionnelle entre le Nord riche et le Sud pauvre tient de moins en moins: Singapour, qui triomphe, est à l'équateur et Moscou, qui décline, au nord de Londres, New York et Tokyo. Cela ne veut cependant pas dire que les évolutions actuelles n'ont pas une cohérence géographique. Au contraire, les régions du monde qui montent bénéficient de tendances «topodynamiques » (c'est-à-dire liées aux forces de localisation) nettement identifiables.

En Amérique, l'axe New York - Los Angeles s'impose clairement et, à l'intérieur de cet axe, le mouvement va nettement de New York vers Los Angeles. En Europe, l'axe Londres - Berlin domine et tout indique que la tendance vers l'est l'emportera sur la tendance vers le sud (le pont avec l'Afrique du Nord se faisant trop lentement) ou même vers le sud-est (l'ex-Allemagne de l'Est, la République tchèque et la Pologne montent plus rapidement que la Croatie ou la Serbie). En Asie, trois axes existent qui partent tous trois de Tokyo. Ce sont l'axe Tokyo - Séoul - Beijing, l'axe Tokyo Shanghai - Canton - Bangkok et l'axe Tokyo - Taiwan - Singapour. Dans les trois cas, le mouvement va vers l'ouest ou le sudouest. En dehors de cela, trois pôles secondaires se développent : le premier, en Inde, dominé par Bombay, le deuxième, en Amérique latine, dominé par São Paulo et le troisième, en Afrique du Sud, dominé par Johannesburg.

\section{MORBIDITÉ DES VILLES RICHES OU MONTANTES : ÉTALEMENT ET TROU DE BEIGNE}

Lorsqu'on aborde les problèmes liés à l'urbanisation actuelle, il convient de nettement distinguer les problèmes qui affectent les villes riches ou montantes de ceux qui touchent les villes pauvres. Dans les villes riches, les problèmes tiennent essentiellement à l'évolution du tissu urbain et à l'usage abusif de l'automobile, l'un et l'autre étant directement reliés. 
L'étalement urbain est particulièrement grave dans les villes riches et cela se traduit par une baisse progressive des densités au centre (ce qui peut donner lieu au phénomène de «trou de beigne» observé en Amérique du Nord) ainsi que par la formation en périphérie des agglomérations de edge cities caractérisées par l'émergence de pôles d'emplois au sein de ce qui était des banlieues dortoirs. En somme, au moment structures, pollution, bruit, congestion, embouteillages, menaces terroristes, etc.) alors même que les ressources fiscales plafonnent (surtout à cause de l'exode des classes moyennes vers la banlieue). La seconde zone située entre les deux rayons jouit d'une situation enviable. Elle attire les investissements industriels, commerciaux et immobiliers ainsi qu'une population nouvelle généralement aisée. Enfin, hors de la

PLUSIEURS VILLES DU TIERS MONDE SERVENT DE PLUS EN PLUS NON PAS DE TREMPLINS, MAIS BIEN DE REFUGES, DE BOUÉES DE SAUVETAGE AU MILIEU DE LA TEMPÊTE CAUSÉE PAR LA POLARISATION DES PRODUCTIONS À L'ÉCHELLE MONDIALE.

où les agglomérations deviennent de plus en plus grosses, elles peuvent devenir aussi de plus en plus informes, leur centre pouvant se marginaliser à cause de la concurrence que lui font les sous-centres de la banlieue. Le concept de centralité doit être totalement revu dans ce nouveau contexte métropolitain. Au plan politique, cela conduit à des remises en question de la notion de citoyenneté et de la place des municipalités existantes dans la dynamique métropolitaine. Les questions de gouvernance revêtent dans ce contexte une importance majeure.

Deux rayons jouent un rôle important dans la compréhension de la nouvelle dynamique urbaine. Un premier rayon situé à quelques kilomètres du centre délimite la zone centrale à l'intérieur de laquelle des baisses de densité et même des phénomènes de «trou de beigne » sont susceptibles d'être observés. Ce rayon correspond à la distance $\mathrm{du}$ centre où les forces d'attraction du centre sont neutralisées par les forces de répulsion liées, entre autres, à la pollution, à la congestion ou à la criminalité du centre. Le second rayon coïncide avec la «marge d'extension » de l'agglomération. Ce rayon est tel que les zones qui sont situées près du périmètre délimité par ce rayon, mais à l'intérieur de ce dernier tirent profit du phénomène de l'étalement urbain, alors que les zones situées près du périmètre délimité par ce rayon, mais à l'extérieur de ce dernier, non seulement ne profitent pas de l'étalement, mais se voient affaiblies du fait de la concurrence croissante que leur livre l'agglomération en expansion.

Du point de vue sociopolitique, en Amérique du Nord, la zone centrale délimitée par le premier rayon est confrontée à des défis croissants (pauvreté, taudification, itinérance, criminalité, usure des infra- marge d'extension, une situation intermédiaire est observée, marquée par un déclin suffisamment lent pour ne pas être dramatique. Ce déclin est tempéré par le fait que les habitants de ces zones nourrissent l'espoir de voir un jour la marge d'extension les englober.

Ce schéma classique en Amérique du Nord est quelque peu différent en Europe occidentale où, traditionnellement, les classes moyennes et supérieures ont beaucoup moins eu tendance à quitter le centre et où les banlieues ont délibérément été aménagées pour accueillir les classes les plus pauvres. Néanmoins, même là, les densités ont tendance à diminuer au centre et les effets de l'étalement urbain sont comparables à ce qui se passe en Amérique du Nord de part et d'autre de la marge d'extension.

Tout cela pose le problème de la gouvernance métropolitaine. Comment faire prévaloir l'intérêt commun métropolitain dans un contexte où les intérêts objectifs des uns et des autres divergent de façon systémique ? Comment concilier les demandes pressantes du centre aux prises avec des problèmes croissants dans un contexte de ressources de plus en plus rares avec le sentiment de quiétude des banlieusards satisfaits de leur prospérité, de leur vie calme et de leurs taxes modérées? Pour les habitants du centre, la vie en ville ne prend tout son sens qu'au centre, tandis que pour les banlieusards, ce n'est pas la ville, mais bien le centre-ville qui fait échec à la vie. Or, le centre peut-il vivre comme centre sans sa périphérie, et la périphérie peut-elle jouir de ses avantages sans que le centre ne joue pleinement son rôle? Voilà toute la question. Il convient de remarquer qu'à aucun moment de la longue histoire de l'urbanisation, cette question n'a été aussi d'actualité.

\section{MORBIDITÉ DES VILLES PAUVRES : SIDA, BIDONVILLES ET REFUGE DE L'INFORMEL}

Parler des problèmes des villes riches contemporaines devient presque gênant quand on songe à ceux des grandes villes du tiers monde. Néanmoins, certaines similitudes existent entre l'évolution des unes et des autres. Tant dans les villes riches que pauvres, on assiste à un étalement urbain généralisé et à une augmentation du transport motorisé, de la pollution et de la congestion ainsi qu'à une difficulté accrue de trouver les formules idéales de gouvernance locale et métropolitaine.

Cela dit, l'ensemble de ces problèmes connaissent une acuité telle dans les villes pauvres que les solutions envisageables dans les pays riches sont le plus souvent hors de portée dans les pays pauvres. Cela tient au manque de ressources, mais aussi au poids des problèmes additionnels posés par le caractère informel d'une grande partie des économies pauvres, par la difficulté de mettre en place un système efficace de perception de taxes locales, par la difficulté compréhensible pour les fonctionnaires locaux très mal rémunérés de résister à la tentation de la corruption et par le fait que la pauvreté favorise grandement la criminalité.

Bien plus, les villes pauvres connaissent une morbidité physique beaucoup plus élevée que les villes riches. Le sida y prolifère de plus en plus; la gestion des eaux usées étant grandement déficiente, les conditions sanitaires sont propices au développement de maladies de toutes sortes. Face à tant de défis démesurés, les autorités locales sont souvent poussées à l'abdication de leurs responsabilités. Le service d'enlèvement des déchets renonce peu à peu à sa mission. Le transport en commun est laissé à l'initiative privée (parfois avec de bons résultats). L'urbanisme laisse place à l'improvisation. Les services policiers se corrompent et les autorités politiques finissent par gérer bien plus leurs propres intérêts que ceux de leurs concitoyens.

Tous ces phénomènes sont décuplés en situation de sur-urbanisation. Actuellement, c'est dans les pays les plus pauvres, tout particulièrement en Afrique, que la croissance de l'urbanisation est la plus rapide. Le taux d'urbanisation de l'Afrique était de $14,8 \%$ en 1950 ; il a atteint 42,2\% en 2000. Cette explosion urbaine se produit alors que l'Afrique, qui compte pour $12,9 \%$ de la population mondiale, ne représente que 3,1 \% du PNB mondial et a un taux de croissance économique inférieur à la moyenne mondiale $(2,74 \%$ comparé à $3,01 \%$ pour le monde entier, au cours de la période 1973-1998). Il est clair que la croissance urbaine dans les pays pauvres est souvent 
plus attribuable au fait que les villes servent de refuges à des populations désespérées qu'à une saine croissance économique.

\section{L'URBAIN COMME REMÈDE AUX MAUX URBAINS}

L'urbanisation n'est ni un problème, ni le fruit d'une politique, c'est un fait lié aux conditions de la mobilité. Chercher à freiner l'urbanisation de façon autoritaire a donné lieu à des atrocités (au Cambodge, en Éthiopie et peut-être même en Chine à l'époque de Mao) sans grand résultat à long terme. L'urbanisation est un fait incontournable dont il faut tirer le meilleur parti. Si souvent la ville fait échec à la vie et si elle est associée par certains à la morbidité, elle est aussi une grande et belle source de vie, de progrès, de convivialité et de sociabilité. La "civitas» demeure le haut lieu de la « civilisation » et l' « urbs », celui de l' «urbanité ».

L'urbanisation offre de magnifiques occasions à ceux qui veulent les saisir. La ville est source d'opportunités, ce qui explique d'ailleurs que la sur-urbanisation soit possible. Elle favorise la mobilité sociale. Elle facilite l'éducation, l'apprentissage, la modernisation, la saine concurrence et le rattachement au reste du monde. Elle met en contact avec la complexité du monde moderne. Elle s'insère dans les grands réseaux de transport et de communications. Elle est porteuse de l'avenir de l'humanité et rien n'indique que le développement des nouvelles technologies de télécommunication puisse remettre en question sa place dans l'évolution mondiale, au contraire.

\section{BIBLIOGRAPHIE}

BAIROCH, Paul (1985). De Jéricho à Mexico: villes et économie dans l'histoire, Paris, Gallimard, Arcades, 708 pages.

BRAUDEL, Fernand (1966). La Méditerranée et le monde méditerranéen à l'époque de Philippe II, Paris, Armand Colin, 2 volumes, 588 et 628 pages.

BRAUDEL, Fernand (1973). Capitalism and Material Life, 1400-1800, New York.

BRAUDEL, Fernand (1977). Afterthoughts on Capitalism and Material Civilization, Baltimore, Md.

BRAUDEL, Fernand (1979). Civilisation matérielle, économie et capitalisme: $15^{e_{-}}$ $18^{e}$ siècle, Paris, Armand Colin, 3 tomes, 544, 600 et 607 pages; traduit sous le titre de Capitalism and Material Life, 14001800, New York, 1973.

DE VRIES, Jan (1976). Economy of Europe in an Age of Crisis, 1600-1750, Cambridge, Cambridge University Press.

DE VRIES, Jan (1984). European Urbanization, 1500-1800, Londres et Cambridge, Mass., Harvard University Press, 398 pages.

HOHENBERG, Paul M. et Lynn HOLLEN LEES (1985). The Making of Urban Europe, 1000-1950, Cambridge (Mass.) et Londres, Harvard University Press, 398 pages; traduit sous le titre de La formation de l'Europe urbaine, 100-1950, Paris, Presses universitaires de France, 1992, 496 pages.

MADDISON, Angus (2001). The World Economy: A Millennium Perspective, Paris, OECD, 385 pages.

TELLIER, Luc-Normand (1997). "A challenge for regional science : revealing and explaining the global spatial logic of economic development », Papers in Regional Science, vol. $76, n^{\circ} 4$, p. 371-384.
TELLIER, Luc-Normand (2001). «Développement, inertie, déterminisme, volontarisme et fatalisme: le défi québécois face à l'évolution spatio-économique mondiale», Organisations et territoires, vol 10, $\mathrm{n}^{\circ} 3$, automne 2001, p. 79-87.

TELLIER, Luc-Normand (2002). Anoeconomics: Exploring the Space-Economic Logic of World History, 1150 pages (à paraître).

\section{Notes}

1. Voir au sujet de l'histoire de l'urbanisation l'ouvrage classique de Bairoch (1985). La synthèse de l'histoire présentée ici diffère, cependant, en partie de la vision développée par cet auteur. Elle découle de Tellier (2002).

2. Même si Braudel n'a jamais tracé ce parallèle entre le début de l'urbanisation et la structure moderne des systèmes urbains, la vision des choses présentée ici est cohérente avec les notions de centre, de semipériphérie et de périphérie chez Braudel (1966, 1973, 1977 et 1979).

3. L'interprétation de l'histoire du développement économique en termes des «corridors topodynamiques » a été proposée par Tellier (1997, 2001 et 2002).

4. C'est cette période qu'a tout particulièrement étudiée Braudel (1966 et 1979). Voir aussi concernant l'Europe urbaine de cette époque de Vries (1976 et 1984) et Hohenberg et Lees (1985). 